\title{
Inventário Rápido de Vespas Sociais em Três Ambientes com Diferentes Vegetações
}

\author{
Newton José de Jesus Silva ${ }^{1}$, Tatiana Aparecida de Morais², Helba Helena Santos-Prezoto ${ }^{3}$ \& Fabio Prezoto $^{1}$
}

1. Universidade Federal de Juiz de Fora, e-mail: newtonecologia@hotmail.com, fabio.prezoto@ufif.edu.br (Autor para correspondência ${ }^{\varpi}$ ). 2. Embrapa Gado de Leite, e-mail: tatianamorais@ig.com.br. 3. Centro de Ensino Superior de Juiz de Fora, e-mail: helba.santos@ig.com.br

\section{EntomoBrasilis 6(2): 146-149(2013)}

\begin{abstract}
Resumo. Apesar de essenciais, trabalhos que objetivem conhecer a riqueza de vespas em meios naturais e agrícolas são incipientes, refletindo em escassez de tempo, recursos e logística adequada para a sua elaboração. Assim, nosso estudo buscou conhecer as espécies de vespas sociais em área com fragmento florestal, plantio de eucaliptos e pomar, além de testar a hipótese de que inventários rápidos, utilizando-se a busca ativa e armadilhas atrativas são estratégias viáveis para amostrar espécies com maior abundância. Foram coletadas 365 vespas sociais, distribuídas em quatro gêneros e 10 espécies, de modo que o pomar registrou maior riqueza específica $(\mathrm{s}=8)$, diversidade $\left(\mathrm{H}^{\prime}=0,5131\right)$ e abundância $(\mathrm{n}=218)$. Tanto Agelaia vicina (Saussure), quanto Polybia ignobilis (Haliday) e Polistes versicolor (Oliver) foram relatadas nas três áreas. O gênero Agelaia contabilizou mais de $70 \%$ dos indivíduos coletados, resultados equivalentes aos de trabalhos conduzidos em fitofisionomias similares, validando a hipótese apresentada. Os inventários constituem-se, ferramentas eficazes na amostragem de espécies de vespas sociais abundantes, refletindo na diminuição de custos e logística em trabalhos de levantamento, contribuindo com ampliação do número de estudos sobre conservação e monitoramento ambiental, além dos relacionados a programas de manejo integrado de pragas.
\end{abstract}

Palavras-chave: Eucalipto; Fragmento Florestal; Polistinae; Pomar.

\section{A Fast Inventory of Social Wasps in Three Environments with Different Vegetation}

Abstract. Although essential, researches that seek to discover the richness of wasps in natural and agricultural environments are incipient, reflecting in shortage of time, resources and adequate logistics for its formulation. Therefore, our study sought to know the species of social wasps in areas with forest fragments, eucalyptus plantations and orchard, in addition to test the hypothesis that fast inventories involving active search and attractive traps are viable strategies for sampling species with greater abundance. We collected 365 social wasps, distributed amongst four genera and 10 species, and the orchard reported the greatest specific richness $(\mathrm{s}=8)$, diversity $\left(\mathrm{H}^{\prime}=0.5131\right)$ and abundance $(\mathrm{n}=218)$. Agelaia vicina (Saussure), Polybia ignobilis (Haliday) and Polistes versicolor (Oliver) were found in the three areas. The genus Agelaia accounted over $70 \%$ of the collected individuals, results equivalent to those carried out in similar works regarding phytophysiognomy, thus validating the presented hypothesis. The inventories are effective tools on sampling abundant social wasps species, reflecting on the decrease of costs and logistics in diversity studies, contributing with an increase of the number of conservation and environmental monitoring research, in addition to the ones related to integrated pest management programs.

Keywords: Eucalyptus; Forest Fragment; Orchard; Polistinae.

$\sqrt{V}$ espas da subfamília Polistinae apresentam distribuição cosmopolita, com ampla representatividade na região tropical, sendo o Brasil considerado o país com a maior diversidade no mundo, com aproximadamente 319 espécies, dispostas em 26 gêneros descritos (Рвеzото et al. 2007), associando-se a diversas atividades nos ambientes naturais e cultivados, como polinização de espécies vegetais, bioindicação da qualidade ambiental e predação de inúmeras espécies de insetos (Souza et al. 2010; РREZoto et al. 2011).

Segundo SANTosetal.(2007), a refinadaligação entre comunidades de vespas e composição vegetacional, possibilita que áreas mais heterogêneas em fitofisionomia, mantenham maior número de espécies destes insetos, pois, a vegetação, influencia suas abundâncias e diversidades diretamente, fornecendo além de alimento e água, fibra vegetal para construção de ninhos e substratos para nidificação. No entanto, sistemas cultivados, menos complexos estruturalmente, apresentam pretensão à proliferação de pragas (RodRIGues et al. 2009), como Heraclides thoas brasiliensis (Roth. \& Jordan), Spodoptera frugiperda (J. E. Smith), Mocis latipes (Guenèe), Diatraea saccharalis (Fabricius), muitas das quais são pertencentes à ordem Lepidoptera, principal fonte proteica para a alimentação de larvas de vespas sociais (РREzoto et al. 2008).
Exemplos recentes sobre diversidade de vespas podem ser encontrados em estudos de Clemente et al. (2012), conduzido em Campo Rupestre e Mata Ciliar; TANAKA JR \& Noll (2011), investigando fragmentos florestais no noroeste de São Paulo; Somavilla \& Köhler (2012), listando vespídeos visitantes de florações em bioma Mata Atlântica no estado do Rio Grande do Sul; Simões et al. (2012), inventariando bioma de Cerrado do sul do estado de Minas Gerais; Ribeiro JR. (2008), realizando levantamento de vespas em eucaliptocultura e AuAD et al. (2010), registrando espécies de vespas em sistema pastoril, ambos no município de Coronel Pacheco, Minas Gerais.

Entretanto, apesar de essenciais, trabalhos que visam conhecer a riqueza de vespas em meios naturais e agrícolas são incipientes, refletindo em alguns casos à escassez de tempo, recursos e logística adequada para a sua elaboração.

Como alternativa, avaliações biológicas rápidas têm sido utilizadas em levantamentos da entomofauna. Assim, este estudo buscou conhecer as espécies de vespas sociais em área com fragmento florestal, plantio de eucaliptos e pomar, além de testar a hipótese de que inventários rápidos, utilizando-se busca ativa e armadilhas atrativas, são estratégias viáveis para amostrar espécies com maior abundância de indivíduos. 


\section{MATERIAL E MÉTODOS}

O estudo foi desenvolvido em uma propriedade rural localizada

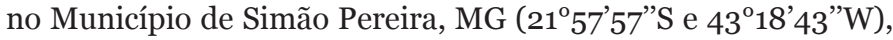
no período de agosto a novembro de 2008 , em áreas contendo plantio de eucaliptos (Eucalyptus grandis W. Hill ex Maiden X E. urophylla ST Blake) com três anos de idade, pomar (Citrus reticulata Blanco (mexerica), Myrciaria jaboticaba (Vell.) (jabuticabeira) e Mangifera indica L. (mangueira) e fragmento florestal (resíduo de Mata Atlântica), mediante coletas mensais.

Para a coleta dos espécimes, dois métodos de amostragem foram utilizados: busca ativa, caracterizada por caminhamentos aleatórios pelas bordas e dentro dos locais de estudo (entre 10:00 e 16:00 h), e armadilhas atrativas (garrafas PET de dois litros com três furos laterais triangulares de $2 \times 2 \times 2 \mathrm{~cm}$ ), abastecidas com três substâncias (suco de maracujá, caldo de sardinha e água), modificado de DE Souza et al. (2011).

Todas as áreas possuíram distribuição das armadilhas por meio de transectos fixos e lineares com $90 \mathrm{~m}$ de comprimento e espaçamento entre as armadilhas de $10 \mathrm{~m}$. Por data de amostragem, somando-se todos os ambientes, foram utilizadas 27 armadilhas, constituindo-se nove por área, e os insetos coletados foram identificados por meio de chaves propostas por RICHARDS (1978) e CARPENTER \& MARQUES (2001), além de comparação com os exemplares da coleção entomológica do Laboratório de Ecologia Comportamental da Universidade Federal de Juiz de Fora (UFJF).

Os dados climatológicos mensais de temperatura $\left({ }^{\circ} \mathrm{C}\right)$ e umidade relativa do ar (\%) foram disponibilizados pelo Laboratório de Climatologia e Análise Ambiental (LabCAA), instalado no Departamento de Geociências do Instituto de Ciências Humanas da UFJF e foram correlacionados através do teste de correlação de Spearman com o número de vespas coletadas.
A existência de diferença entre as áreas de acordo com o número de indivíduos coletados foi calculada pelo teste do Qui-quadrado $\left(\chi^{2}\right)$. Para análise de diversidade, foi utilizado o índice de Shannon-Wiener (H'), e correlação entre as variáveis climáticas e insetos capturados, o coeficiente de Spearman (rs), executados pelo programa BioEstat v5.o (AyrEs et al. 2007). A avaliação da dominância e equidade nos três ambientes, foi feita pelo índice de dominância $\left(I_{\mathrm{s}}\right)$ e equitabilidade $\left(\mathrm{E}_{\mathrm{D}}\right)$ de Simpson, utilizandose o software DivEs - Diversidade de Espécies v. 2.0 (RoDRIGUES 2005).

\section{RESULTADOS E DISCUSSÃO}

Ao fim do estudo, foram registrados quatro gêneros (Agelaia, Polistes, Polybia e Protopolybia) e 10 espécies, totalizando 365 indivíduos coletados, apresentando o pomar, maior riqueza específica $(\mathrm{s}=8)$ e diversidade $\left(\mathrm{H}^{\prime}=0,5131\right)$, seguido, respectivamente, pelo plantio de eucaliptos $\left(\mathrm{s}=6 ; \mathrm{H}^{\prime}=0,4887\right)$ e fragmento florestal ( $\left.\mathrm{s}=5 ; \mathrm{H}^{\prime}=0,3856\right)$ (Tabela 1$)$.

O pomar diferiu significativamente em número de vespas amostradas $(\mathrm{n}=218)$, com o plantio de eucaliptos $\left(\mathrm{n}=67 ; \chi^{2}\right.$ $=145,2072 ; \mathrm{p}<0,05)$ e o fragmento florestal $\left(\mathrm{n}=80 ; \chi^{2}=\right.$ 190,3286; $\mathrm{p}<$ 0,05), não sendo o mesmo encontrado entre plantio de eucaliptos e fragmento florestal $\left(\chi^{2}=49,1944 ; p=0,1270\right)$.

A superioridade tanto da riqueza e diversidade, quanto da abundância de vespas sociais no pomar, pode estar relacionadas ao período de frutificação das espécies cultivadas nesta área (jabuticabas e mexericas), coincidente com os meses de coleta. As vespas possuem comportamento oportunista, e são atraídas para ambientes que forneçam maior quantidade de recursos como água e alimento, o que faz com que explorem ambientes cultivados, menos complexos em fitofisionomia (SANTos et al. 2009; De Souza et al. 2010; Brugger et al. 2011). As espécies Agelaia vicina (Saussure), Polybia ignobilis (Haliday) e Polistes versicolor (Oliver) foram relatadas em todos os locais de estudo

Tabela 1. Espécies de vespas sociais (Polistinae), coletadas na zona rural do município de Simão Pereira, MG, no período de agosto a novembro de 2008, em diferentes áreas de amostragem. - Espécies Ausentes; B.A.= Busca Ativa; M.= Armadilhas com Maracujá; S.= Armadilhas com Sardinha.

\begin{tabular}{|c|c|c|c|c|c|c|c|c|c|}
\hline \multirow{3}{*}{ Gêneros e Espécies } & \multicolumn{3}{|c|}{ Área de Pomar } & \multicolumn{3}{|c|}{ Plantio de Eucaliptos } & \multicolumn{3}{|c|}{ Fragmento Florestal } \\
\hline & \multicolumn{2}{|c|}{ Armadilhas } & \multirow{2}{*}{ B. A. } & \multicolumn{2}{|c|}{ Armadilhas } & \multirow{2}{*}{ B. A. } & \multicolumn{2}{|c|}{ Armadilhas } & \multirow{2}{*}{$\begin{array}{c}\text { B. A. } \\
-\end{array}$} \\
\hline & M. & S. & & M. & s. & & M. & s. & \\
\hline Agelaia sp2 & $6(1,64 \%)$ & $5(1,37 \%)$ & $1(0,27 \%)$ & - & - & - & $7(1,92 \%)$ & $22(6,03 \%)$ & $1(0,27 \%)$ \\
\hline Agelaia vicina (Saussure) & $24(6,58 \%)$ & $81(22,19 \%)$ & $23(6,30 \%)$ & $15(4,11 \%)$ & $20(5,48 \%)$ & $6(1,64 \%)$ & $8(2,19 \%)$ & $36(9,86 \%)$ & $2(0,55 \%)$ \\
\hline Polistes sp1 & - & - & $8(2,19 \%)$ & - & - & - & - & - & - \\
\hline
\end{tabular}

\begin{tabular}{|c|c|c|c|c|c|c|c|c|c|}
\hline Polistes billardieri Fabricius & - & - & - & $2(0,55 \%)$ & - & - & - & - & - \\
\hline Polistes versicolor (Oliver) & $1(0,27 \%)$ & - & $1(0,27 \%)$ & $4(1,10 \%)$ & - & - & $2(0,55 \%)$ & - & - \\
\hline Polybia ignobilis (Haliday) & $5(1,37 \%)$ & - & $2(0,55 \%)$ & $2(0,55 \%)$ & - & - & $1(0,27 \%)$ & - & - \\
\hline Polybia platycephala Richards & - & - & $3(0,83 \%)$ & $2(0,55 \%)$ & - & - & - & - & - \\
\hline
\end{tabular}

\begin{tabular}{|c|c|c|c|c|c|c|c|c|c|}
\hline Polybia scutellaris (White) & $1(0,27 \%)$ & - & - & - & - & - & - & - & - \\
\hline Polybia sericea (Oliver) & $18(4,93 \%)$ & $1(0,27 \%)$ & $\begin{array}{c}38 \\
(10,41 \%)\end{array}$ & $14(3,84 \%)$ & - & $2(0,55 \%)$ & - & - & - \\
\hline
\end{tabular}

Protopolybia sp1 $(10,41 \%)$

6

( $\Sigma)$ Abundancia Metodologia

55

87

76

39

20

$5 \quad 2$

5 
(Tabela 1), demostrando capacidade adaptativa aos diferentes níveis de interferência antrópica.

Os valores encontrados para o índice de dominância $\left(I_{s}\right)$ por área de estudo foram: pomar $\left(I_{s}=0,1509\right)$, plantio de eucaliptos $\left(I_{s}=0,1063\right)$ e fragmento florestal $\left(I_{s}=0,1646\right)$, resultados que refletem a maior proporção de espécimes de vespas $A$. vicina no fragmento florestal, além do menor número de espécies registradas neste ambiente.

A elevada frequência de indivíduos do gênero Agelaia, 70\% (n = 257) dentre os insetos coletados no total dos três locais de estudo, corrobora os resultados encontrados em trabalhos como Souza \& Prezoto (2006) em floresta estacional semidecidual, no município de Barroso, MG, os quais registraram A. vicina como a espécie constante; RiBEIRo JR. (2008) em plantio de eucaliptos em Coronel Pacheco, MG, com mais de 90\% das vespas capturadas pertencentes a este gênero; e AUAD et al. (2010), inventariando sistema pastoril no mesmo município, com $63,42 \%$ das vespas amostradas pertencentes as espécies Agelaia multipicta (Haliday) e A. vicina.

A prevalência deste gênero tem relação com a grande dimensão de suas colônias, podendo conter mais de um milhão de indivíduos (Zucchi et al. 1995; Hunt et al. 2001), o que significa maior número de operárias em forrageio e probabilidade de captura.

Comparando-se o presente estudo com trabalhos conduzidos em ambientes com fitofisionomia próxima, este alcançou similaridade e mesmo equivalência em riqueza específica no caso do bioma de
Mata Atlântica, demonstrando confiabilidade na amostragem das espécies com maior abundância, além de facilidade e praticidade na metodologia adotada (Tabela 2).

Por meio da análise da equidade, revelou-se menor índice para o fragmento florestal $\left(\mathrm{E}_{\mathrm{D}}=0,5278\right)$, sucedido por plantio de eucaliptos $\left(\mathrm{E}_{\mathrm{O}}=0,5623\right)$ e pomar $\left(\mathrm{E}_{\mathrm{D}}=0,5812\right)$, sendo esta uniformidade explicada pela menor probabilidade de localização das espécies de vespas e suas colônias em habitas com densa vegetação, tornando-as mais crípticas nestes ambientes, e aumento na dificuldade de sua amostragem em regiões com subbosque desenvolvido (Clemente et al. 2012).

Não foi encontrada correlação significativa $(\mathrm{RS}=0,1875 ; \mathrm{p}$ $=0,2465)$ entre a temperatura $\left(21,5 \pm 2,97^{\circ} \mathrm{C}\right)$ e o número de vespas coletadas, assim como entre estas $(\mathrm{RS}=-0,0351 ; \mathrm{p}=$ o,8298) e a umidade relativa do ar $(57,00 \pm 19,93 \%)$, devido possivelmente ao período transicional entre as estações fria/ seca (agosto e setembro) e quente/úmida (outubro e novembro), de modo que as variações entre os valores registrados para os dados climatológicos nesses meses são menos acentuadas quando comparadas às de meses com maior estabelecimento das condições climáticas.

Os resultados obtidos confirmam a hipótese de que os inventários constituem-se ferramentas eficazes na amostragem de espécies de vespas sociais com maior abundância, auxiliando na obtenção de dados com menor gasto de tempo, além de proporcionar diminuição de custos e logística.

Tabela 2. Comparação entre riqueza especifica no presente estudo e outros trabalhos da literatura. *Excluídas armadilhas de dossel.

\begin{tabular}{|c|c|c|c|c|}
\hline Ambiente & Metodologia de coleta & $\begin{array}{c}\text { Tempo de } \\
\text { Execução (meses) }\end{array}$ & $\begin{array}{l}\text { Riqueza } \\
\text { Especifica }\end{array}$ & Referência \\
\hline Eucaliptocultura & Busca Ativa e Armadilhas (garrafas pet)* & 12 & 16 & DE Souza et al. (2011) \\
\hline Mata Atlântica (Neves Paulista) & $\begin{array}{l}\text { Coleta ativa com utilização de líquido } \\
\text { atrativo }\end{array}$ & 6 & 12 & Gomes \& Noll (2009) \\
\hline Eucaliptocultura & Busca Ativa e Armadilhas (garrafas pet) & 12 & 12 & RiBEIRO JR. (2008) \\
\hline Pomar & Busca Ativa e Armadilhas (garrafas pet) & 13 & 9 & SANTOS (1996) \\
\hline Pomar & Busca Ativa e Armadilhas (garrafas pet) & 4 & 8 & Esse estudo \\
\hline Eucaliptocultura & Busca Ativa e Armadilhas (garrafas pet) & 4 & 6 & Esse estudo \\
\hline Mata Atlântica (Paulo de Faria) & $\begin{array}{l}\text { Coleta ativa com utilização de líquido } \\
\text { atrativo }\end{array}$ & 6 & 7 & Gomes \& Noll (2009) \\
\hline Mata Atlântica (Pindorama) & $\begin{array}{l}\text { Coleta ativa com utilização de líquido } \\
\text { atrativo }\end{array}$ & 6 & 6 & Gomes \& Noll (2009) \\
\hline Mata Atlântica & Busca Ativa e Armadilhas (garrafas pet) & 4 & 5 & Esse estudo \\
\hline Mata Atlântica & $\begin{array}{l}\text { Busca Ativa, Armadilhas Atrativas e Busca } \\
\text { pontual }\end{array}$ & 12 & 5 & Clemente (2009) \\
\hline
\end{tabular}

\section{REFERÊNCIAS}

Auad, A.M., C.A. Carvalho, M.A. Clemente \& F. Prezoto, 2010. Diversity of social wasps in a silvipastoral system. Sociobiology, 55: 627-636.

Ayres, M., M. Ayres Jr. \& A.A.S. dos Santos, 2007. BioEstat. Versão 5.o, Software e Manual para o usuário. Disponível em: <http://www.mamiraua.org.br/downloads/programas $>$.

Brugger, B.P., L.S.S. Araújo, A.R. de Souza \& F. Prezoto, 2011. Social wasps (Synoeca cyanea) damaging Psidium sp. (Myrtaceae) fruits in Minas Gerais state, Brazil. Sociobiology, 57: 533-535.

Carpenter, J.M. \& O.M. Marques, 2001. Contribuição ao estudo dos vespídeos do Brasil (Insecta, Hymenoptera, Vespoidae, Vespidae). Cruz das Almas, Universidade Federal da Bahia. Publicações digitais, Volume 2. Universidade Federal da Bahia.

Clemente, M.A., 2009. Vespas sociais (Hymenoptera, Vespidae) do Parque Estadual do Ibitipoca-MG: estrutura, composição e visitação floral. Dissertação (Mestrado em Comportamento e
Biologia Animal: Ciências Biológicas) - Universidade Federal de Juiz de Fora. 79p.

Clemente, M.A., D. Lange, K. Del-Claro, F. Prezoto, N.R. Campos \& B.C. Barbosa, 2012. Flower-visiting social wasps and plants interaction: Network Pattern and Environmental Complexity, Psyche: A Journal of Entomology, 2012: 1-10.

De Souza, A.R., D. Venâncio \& F. Prezoto, 2010. Social wasps damaging fruit of Myrciaria sp. Sociobiology, 55: 297-299.

De Souza, A.R., D.F.A. Venâncio, J.C. Zanuncio \& F. Prezoto, 2011. Sampling methods for assessing social wasps species diversity in a eucalyptus plantation. Journal of Economic Entomology, 104: 1120-1123.

Gomes, B. \& F.B. Noll, 2009. Diversity of social wasps (Hymenoptera, Vespidae, Polistinae) in three fragments of semideciduous seasonal forest in the northwest of São Paulo State, Brazil. Revista Brasileira de Entomologia, 53: 428431.

Hunt, J.H., S. O’Donnell, N. Chernoff \& C. Brownie, 2001. Observations on two neotropical swarn-founding wasps Agelaia yepocapa and Agelaia panamaensis (Hymenoptera: 
Vespidae). Annals of the Entomological Society of American, 4: 555-562.

Prezoto, F., A.R. De Souza, H.H. Santos-Prezoto, N.J.J. Silva \& V.Z. Rodrigues, 2011. Estudos comportamentais em vespas sociais: da história natural a aplicação, p. 87-91. In: TorezanSilingardi, H.M. \& V. Stefani (Org.). Etologia 2011: temas atuais em etologia e Anais do XXIX Encontro Anual de Etologia. Uberlândia, Composer, 257p.

Prezoto, F., C. Ribeiro Jr, D.L. Guimarães \& T. Elisei, 2008. Vespas sociais e o controle biológico de pragas: atividade forrageadora e manejo das colônias, p. 413-427. In: Vilela, E.F., I.A. Dos Santos, J.H. Schoereder, J.E. Serrão, L.A.O. Campos \& J. Lino-Neto (Org.). Insetos sociais: da biologia a aplicação. Viçosa, Editora da UFV, 442p.

Prezoto, F., C. Ribeiro Jr., S.A.O. Cortes \& T. Elisei, 2007. Manejo de vespas e marimbondos em ambiente urbano, p. 123-126. In: Pinto, A.S., M.M. Rossi, \& E. Salmeron (Org.). Manejo de Pragas Urbanas. Piracicaba, CP2, 234p.

Ribeiro Jr, C., 2008. Levantamento das espécies de vespas sociais (Hymenoptera, Vespidae) em eucaliptocultura. Dissertação (Mestrado em Comportamento e Biologia Animal: Ciências Biológicas) - Universidade Federal de Juiz de Fora.

Richards, O.W., 1978. The social wasps of the Americas, excluding the Vespinae. London, British Museum Natural History, 580 .

Rodrigues, W.C., 2005. DivEs - Diversidade de Espécies. Versão 2.0, Software e Guia do usuário. Disponível em: $<$ http://www.ebras.bio.br/dives $>$.

Rodrigues, W.C., P.C.R. Cassino, M.V. Spolidoro \& R. SilvaFilho, 2009. Insetos sugadores (Sternorrhyncha) em cultivo orgânico de tangerina cv. Poncã (Citrus reticulata Blanco): diversidade, constância, frequência e flutuação populacional. EntomoBrasilis, 2: 42-48. Disponível em: <http://www.periodico.ebras.bio.br/ojs >

Santos, B.B, 1996. Ocorrência de vespídeos sociais (Hymenoptera, Vespidae) em pomar em Goiânia, Goiás, Brasil. Agrárias, 15: 43-46.
Santos, G.M.M., C.C. Bichara Filho, J.J. Resende, J.D. Cruz \& O.M. Marques, 2007. Diversity and community structure of social wasps (Hymenoptera, Vespidae) in three ecosystems in Itaparica Island, Bahia State, Brazil. Neotropical Entomology, 36: $180-185$.

Santos, G.M.M., J.D. da Cruz, O.M. Marques \& N. Gobbi, 2009. Diversidade de vespas sociais (Hymenoptera: Vespidae) em áreas de Cerrado na Bahia. Neotropical Entomology, 38: $317-320$.

Simões, M.H., M.D. Cuozzo \& F.A. Frieiro-Costa, 2012. Diversity of social wasps (Hymenoptera, Vespidae) in Cerrado biome of the southern of the state of Minas Gerais, Brazil. Iheringia, 3: 292-297.

Somavilla, A. \& A. Kohler, 2012. Preferência floral de vespas (Hymenoptera: Vespidae) no Rio Grande do Sul, Brasil. Entomobrasilis, 5: 21-28. Disponível: <http://www.periodico. ebras.bio.br/ojs/index.php/ebras/article/view/152>.

Souza, M.M. \& F. Prezoto, 2006. Diversity of social wasps (Hymenoptera: Vespidae) in semideciduos forest and cerrado (savanna) regions in Brazil. Sociobiology, 47: 135-147.

Souza, M.M., J. Louzada, J.E. Serrao \& J.C. Zanuncio, 2010. Social wasps (Hymenoptera: Vespidae) as indicators of conservation degree of Riparian Forests in Southeast Brazil. Sociobiology, 56: 387-396.

Tanaka Junior, G.M. \& F.B. Noll, 2011. Diversity of social wasps on semideciduous seasonal forest fragments with different surrounding matrix in Brazil. Psyche: A Journal of Entomology, 2011: 1-8.

Zucchi, R., S. Sakagami, F.B. Noll, M.R. Mechi, S. Mateus, M.V. Baio \& S.N. Shima, 1995. Agelaia vicina, a swarm-founding polistine with the largest colony size among wasps and bees (Hymenoptera: Vespidae). The New York Entomological Society, 103: 129-137.

\section{Recebido em: 17/12/2012}

Aceito em: 17/05/2013

\section{Como citar este artigo:}

Silva, N.J.J., T.A. Morais, H.H. Santos-Prezoto \& F. Prezoto, 2013. Inventário Rápido de Vespas Sociais em Três Ambientes com Diferentes Vegetações. EntomoBrasilis, 6(2): 146-149.

Acessível em: http://www.periodico.ebras.bio.br/ojs/index.php/ebras/article/view/303. doi:10.12741/ebrasilis.v6i2.303
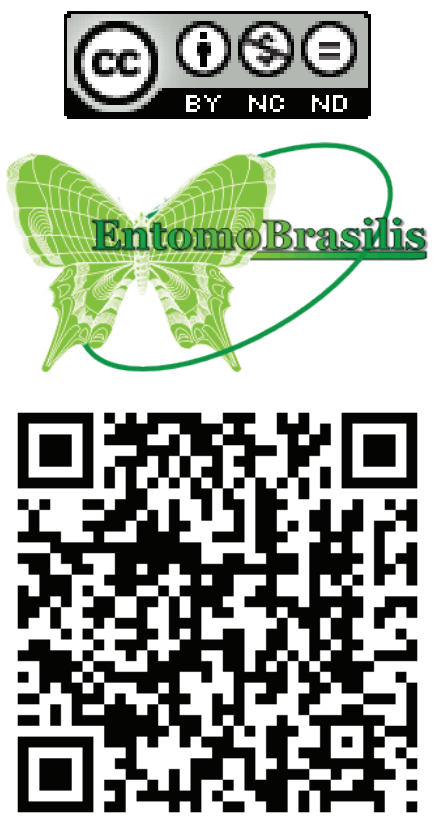